OPEN ACCESS

Edited by:

Hari S. Sharma,

Uppsala University, Sweden

Reviewed by:

Seaab Imad Sahib,

University of Arkansas, United States

Jose Vicente Lafuente,

University of the Basque Country,

Spain

*Correspondence:

Jianwei LV

Ivjianwei@renji.com

Specialty section:

This article was submitted to

Neural Technology,

a section of the journal

Frontiers in Neuroscience

Received: 20 October 2020 Accepted: 05 February 2021

Published: 26 February 2021

Citation:

Gu Y, LV T, Jiang C and Lv J (2021) Neuromodulation of the

Pudendal Nerve Assisted by 3D

Printed: A New Method

of Neuromodulation for Lower Urinary

Tract Dysfunction.

Front. Neurosci. 15:619672.

doi: 10.3389/fnins.2021.619672

\section{Neuromodulation of the Pudendal Nerve Assisted by 3D Printed: A New Method of Neuromodulation for Lower Urinary Tract Dysfunction}

\author{
Yinjun Gu, Tingting Lv, Chen Jiang and Jianwei $L v^{*}$ \\ Department of Urology, Renji Hospital, Shanghai Jiao Tong University School of Medicine, Shanghai, China
}

Electrical stimulation of peripheral nerves by implanted electrodes is an effective treatment for certain pelvic floor diseases. As well as intravesical electrical stimulation, this predominantly includes stimulation of the sacral nerve, tibial nerve, and pudendal nerve. The pudendal nerve is one of the main nerves that stimulate pelvic floor muscles, external urethral meatus, and the anal sphincter and pelvic organs, and it may have effects on frequent urination, urgency, dysuria, and perineal pain. It is difficult to locate because of its anatomical course, however, leading to difficulties fixing the electrode, which increases the difficulty of pudendal nerve electrical stimulation in clinical practice. In the current study 3D printed navigation was used to solve these problems. Combined with autopsy data and patient pelvic and nerve data, a personalized design was generated. Neural modulation of the pudendal nerve was achieved by implanting the lead with the guidance of 3D printed navigation. 3D printed navigation can maximize the phase II conversion rate, reduce the difficulty of surgery, shorten the operation time, reduce damage to additional organs and blood vessels, and increase the accuracy of electrode implantation, and it can be performed while the patient is awake. It is an accurate, reversible, efficient, and minimally invasive surgery.

\section{Keywords: 3D printing, nerve stimulation, pudendal nerve, image fusion, sacral nerve}

\section{INTRODUCTION}

Neuromodulation is an emerging medical field, and it has been shown to be an effective treatment for a variety of pelvic floor diseases and other diseases such as overactive bladder (Salima et al., 2017), interstitial cystitis (Han et al., 2018), and neurogenic bladder (Kessler et al., 2010). The neural regulation of pelvic floor diseases mainly utilizes sacral nerve regulation (Zhang et al., 2019). Sacral neuromodulation therapy was proposed by Schmidt et al. (1979) for the treatment options of lower urinary tract functional disorders. In 2012, China officially launched a large-scale promotion of SNM therapy. In the past 5 years, nearly 60 centers in China have carried out SNM clinical studies, and there are also several clinical articles published in China that report the efficacy and prognosis of SNM (Chen et al., 2014; Zhang et al., 2017). Researchers are also exploring other pelvic floor and peripheral nerves as new regulatory targets to achieve better efficacy and reduce associated 
complications, such as the pudendal nerve (Marinkovic et al., 2015) and the tibial nerve (Bhide et al., 2020). The pudendal nerve is one of the main nerves that stimulates pelvic floor muscles, external urethral meatus, the anal sphincter, and pelvic organs, and it is composed of nerve fibers emanating from S2 to S4 nerve roots. It is a mixed nerve containing somatic and autonomic nerve fibers. Because the pudendal nerve has no bony localization mark, so its surgical localization and puncture are problematic.

Reitz et al. (2007) described a new method of pudendal nerve puncture from the dorsal direction from topographical and anatomical perspectives. They reported that the average insertion point of the needle was $14 \mathrm{~cm}$ in the greater trochanter of the femur, $9 \mathrm{~cm}$ above the ischial tuberosity, and $6 \mathrm{~cm}$ lateral to the gluteus. This enables the needle to have a large area of contact with the pudendal nerve, resulting in greater stimulation efficacy and lower stimulation intensity. Jottard et al. (2017) described a minimally invasive transbuttock endoscopic method for implanting pudendal electrodes for neuromodulation under full visual control. Konschake et al. (2017) accurately and reliably identified the pudendal nerve using laparoscopic techniques, and developed a direct, reliable, and minimally invasive pudendal neuroendoscopic surgical method relying on laparoscopic techniques.

The above-described methods are complex and require a precise, reversible, efficient, and minimally invasive method to puncture and localize the pudendal nerve. Three-dimensional printing technology is being used increasingly in modern medicine in various disciplines, including orthopedics (Lal and Patralekh, 2018) and maxillofacial surgery (Han et al., 2019), and Morrison et al. (2019) have developed a 3D printed device for cranial nerve stimulation in mice (Morrison et al., 2019). Yinjun et al. (2016) and Zhang et al. (2018), respectively, proposed the application of 3D printed technology in sacral neuromodulation, so as to reduce the difficulty of surgery and increase the effect of surgery. In the current study, 3D printed navigation was used to meet the aforementioned design requirements. Autopsy data were combined with pelvic and neurological patient data to achieve personalized procedural design. Neural modulation of the pudendal nerve was achieved by implanting the lead with the guidance of 3D printed navigation.

\section{MATERIALS AND METHODS}

\section{Acquisition of Underlying Data}

The pudendal nerve is composed of nerve fibers arising in the S2-S4 nerve roots, and runs in the pudendal canal and accompanies the internal pudendal artery. From the sacral plexus, the pudendal nerve travels between the piriformis muscle and the coccygeus muscle, then passes through the greater sciatic foramen into the pelvis, spans the ischial spine, passes between the sacrospinous ligament and the sacrotuberous ligament, and terminates at the perineum. Inside the pudendal canal, which is formed by the fascia, the pudendal nerve usually gives rise to three branches: the inferior rectal nerve, the perineal nerve, and the dorsal penile/clitoral nerve (Pirro et al., 2009; Figure 1).
Two days before surgery, a four-point fixed positioning marker was placed on the patient's body surface on the dorsal side of the sacrum, and marker points were marked with gentian violet to prevent bias in guide template placement. A plain computed tomography scan of the sacrum was conducted first using a UCT S-160 scanner (United Imaging, Shanghai, China). The scanning parameters used were tube potential $120 \mathrm{kV}$, tube current $200 \mathrm{~mA}$, matrix, $512 \times 512$, field of view $150 \mathrm{~mm}$, slice thickness $1.5 \mathrm{~mm}$, spacing $1 \mathrm{~mm}$, and pitch 0.9375 , and $3 \mathrm{D}$ bone reconstruction was performed after scanning via a bone reconstruction algorithm. Magnetic resonance imaging (MRI) of the sacral and pudendal nerves was then conducted with a Signa HDXT 3.0T MRI scanner (General Electric, United States), using an 8-channel cardiac coil with the patient in the dorsal position and the foot advanced. Neuroimaging was performed using a $2 \mathrm{D}$, thin-section, cross-sectional, fastspin echo, fat suppression sequence (Axial T2 WI-FSE iDEAL). The scanning parameters were field of view $320 \mathrm{~mm}$, matrix $320 \times 224$, repetition time $5380 \mathrm{~ms}$, echo time $68 \mathrm{~ms}$, echo train length 16, bandwidth $50 \mathrm{~Hz}$, slice thickness $2 \mathrm{~mm}$, spacing $0 \mathrm{~mm}$, and number of excitations 1 . The scanning range for continuous acquisition was from the lower edge of the L5 vertebral body to the lower edge of the S5 vertebral body (Figure 2).

\section{Navigation Template Design and 3D Printed}

Two methods were used to locate the pudendal nerve, autopsy studies and patient-based procedures. Autopsy studies were used to identify the correct position for the needle to ensure the largest contact area with the pudendal nerve. Puncture of the ischial tuberosity on the same side resulted in a larger contact area between the needle and the pudendal nerve (Figure 3). Based on puncture navigation derived from the individual patient's data, the patient's unique pelvic floor anatomy was obtained by fusing sacrum computed tomography imaging and pelvic floor nerve MRI, and the puncture needle path was designed with the requirement of maximum contact area between the puncture needle and the pudendal nerve (Figures 4-6). In either method, the location of the pudendal nerve should be combined with the patient's own imaging data. So 3D printed navigation templates are personalized designs.

\section{Procedure}

A standardized sacral nerve puncture procedure (Expert Consensus Writing Group on Clinical Application of Sacral Neuromodulation, 2017) was used throughout the entire study. The procedure was conducted as follows: (1) Place the patient in a prone position on a digital subtraction machine tool, with the leg slightly elevated and the knee joint bent to ensure toe suspension. (2) Disinfect and drape the sacrococcyx and hip surgical fields and expose the anal region and feet. (3) Match the 3D printed navigation template with the preoperative positioning markers, and perform local anesthesia with reference to the template puncture point. (4) Perform the puncture in accordance with the $3 \mathrm{D}$ printed navigation template, and 

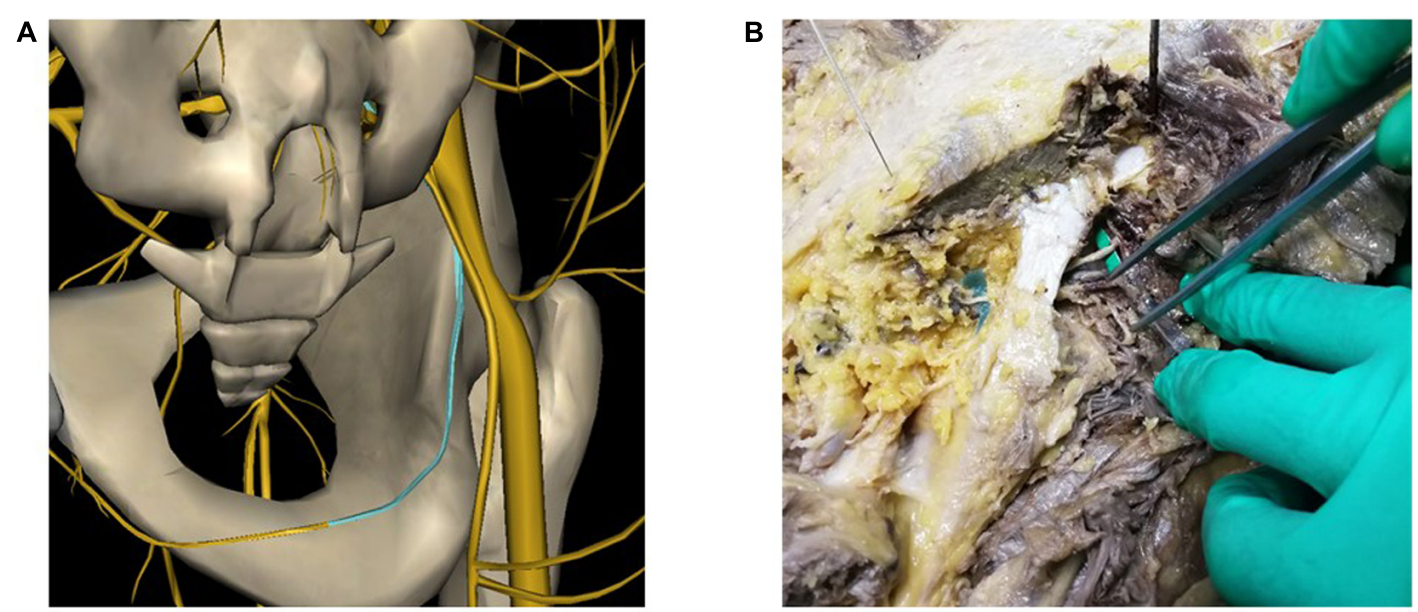
a. 3D simulation of pudendal nerve anatomy
b. Pudendal nerve autopsy diagram

FIGURE 1 | Anatomic localization of the pudendal nerve. (A) 3D simulation of pudendal nerve anatomy. (B) Pudendal nerve autopsy diagram.
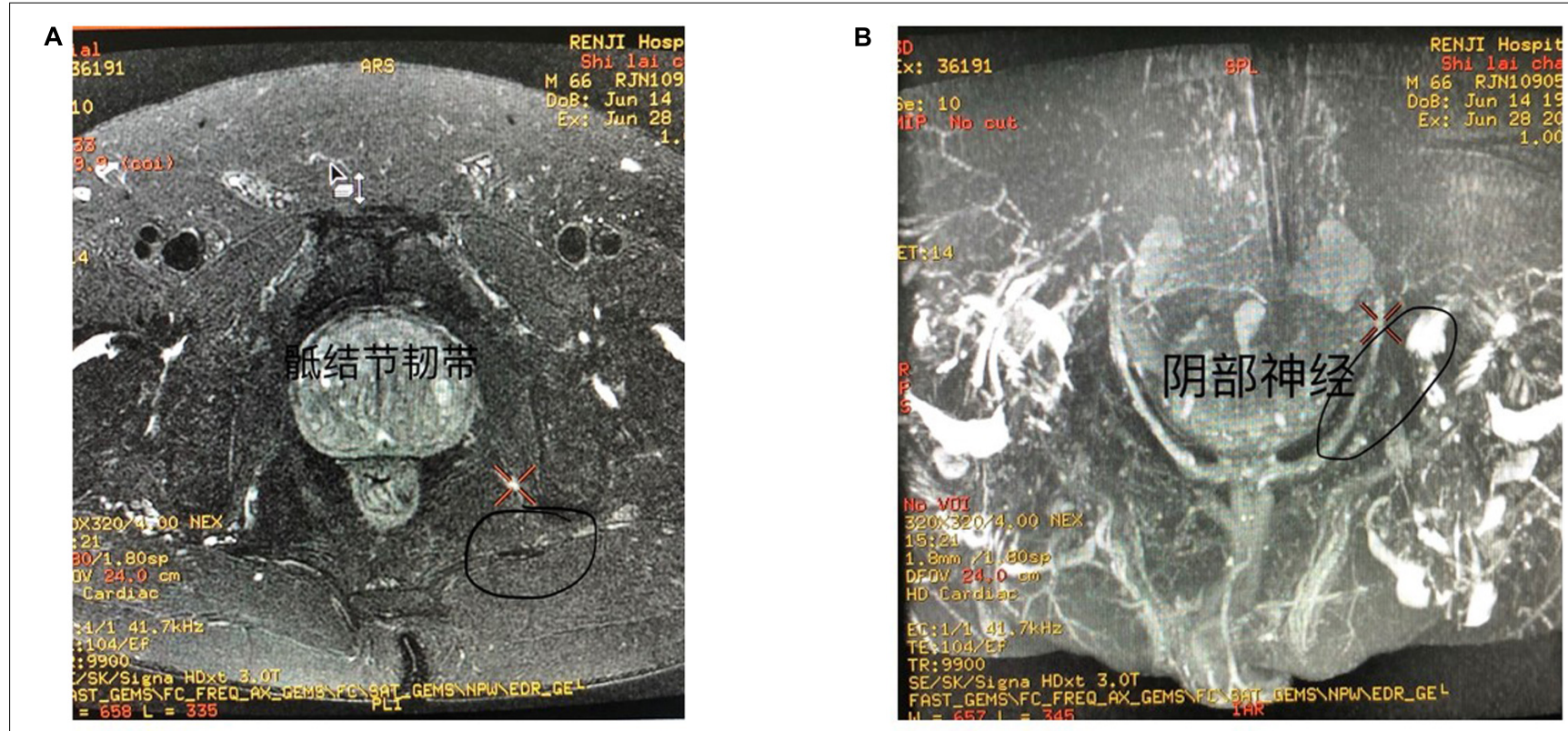

a. MRI images of the pudendal nerve and sacrotuberous ligament in transverse position

b. MRI image of coronal position of pudendal nerve

FIGURE 2 | MRI image of pudendal nerve. (A) MRI images of the pudendal nerve and sacrotuberous ligament in transverse position. (B) MRI image of coronal position of pudendal nerve.

observe the distance between the puncture needle and the ischial tuberosity $45^{\circ}$ below the digital subtraction machine and adjust it. (5) When the needle position is satisfactory, test the patient's motor and sensory responses to determine whether the access site is correct. (6) After confirming that the position of the needle is correct, remove the needle, insert the depth indicator needle, place the electrode introducer sheath containing the dilator along the depth indicator needle, and observe the position of the sheath $45^{\circ}$ on the $\mathrm{x}$-ray side, so that a "breakthrough feeling" is appropriate. (7) Remove the 

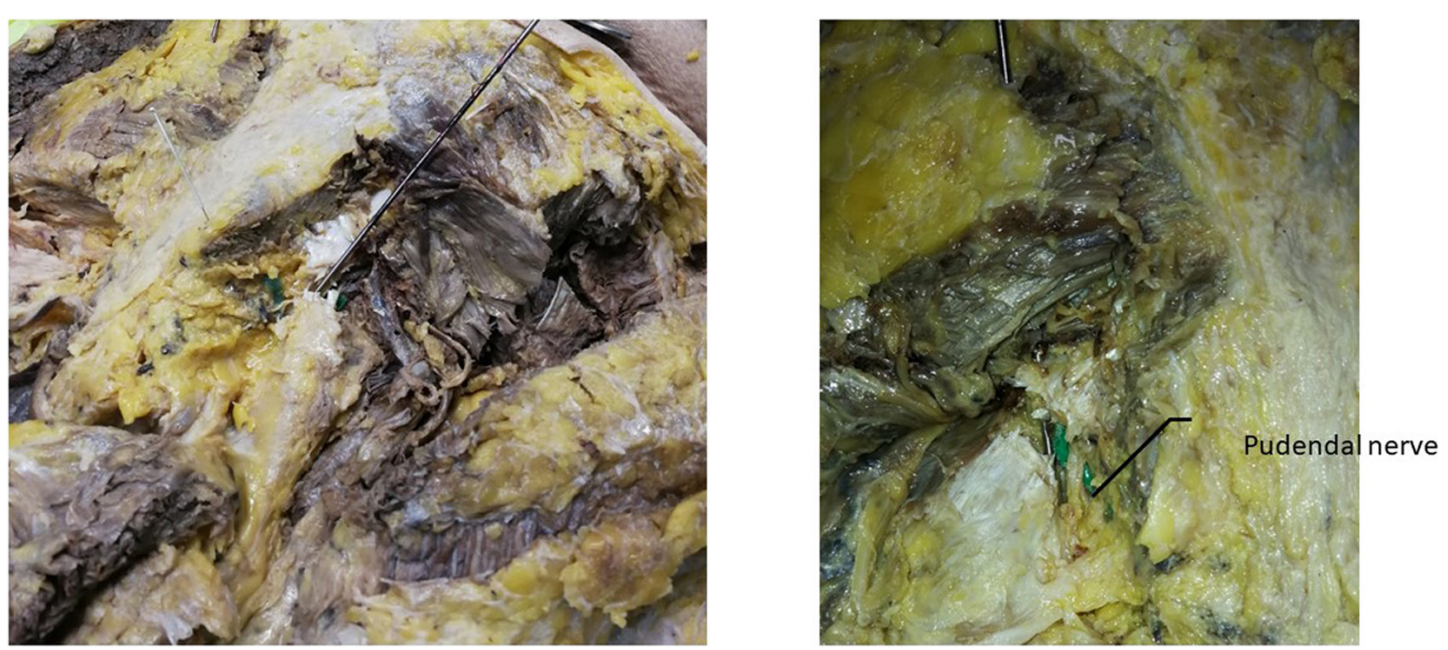

FIGURE 3 | ldeal site for a postmortem puncture.

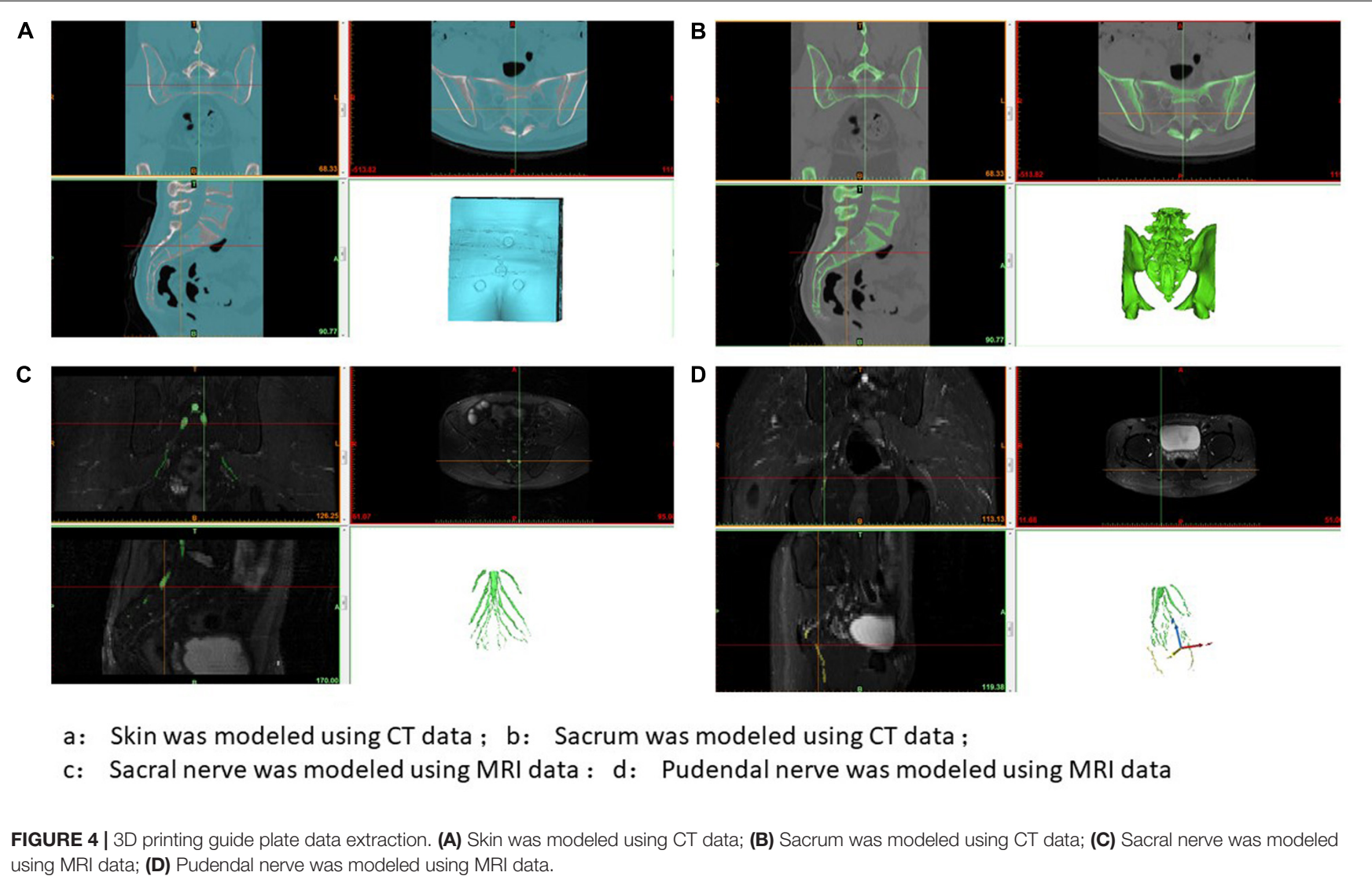

introducer sheath and depth indicator needle, leaving only the dilator. (8) Implant the self-fixing electrode. Once the test is satisfied, keep the barbed electrode still, remove the sheath, and fix the electrode. (9) Make a 3-cm incision in the outer upper right buttock for the permanent stimulator to be implanted into. Use a subcutaneous tunneler to draw out the barbed electrode tail end, connect the percutaneous extension, and guide the percutaneous extension out of the skin of the opposite buttock through the subcutaneous tunnel (10). Test each shock response and close the buttock incision (Figures 7, 8). The protocol was approved by the Shanghai Jiao Tong University School of Medicine.

During the operation, the sacral nerve electrode and pudendal nerve electrode were placed simultaneously. The degree 

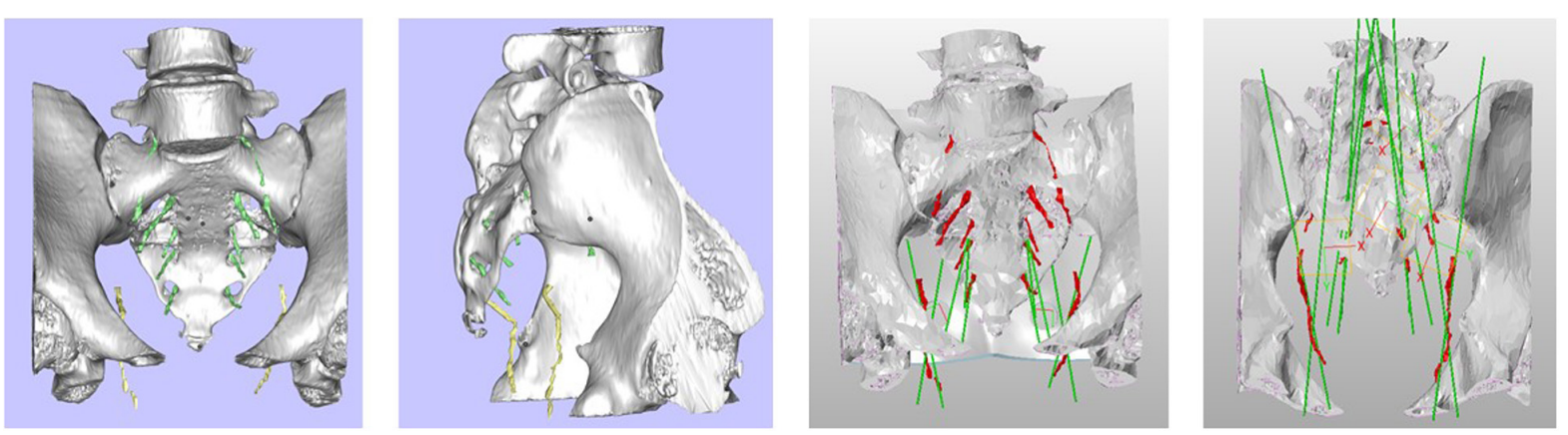

FIGURE 5 | 3D printing guide plate CT and MRI data fusion modeling.
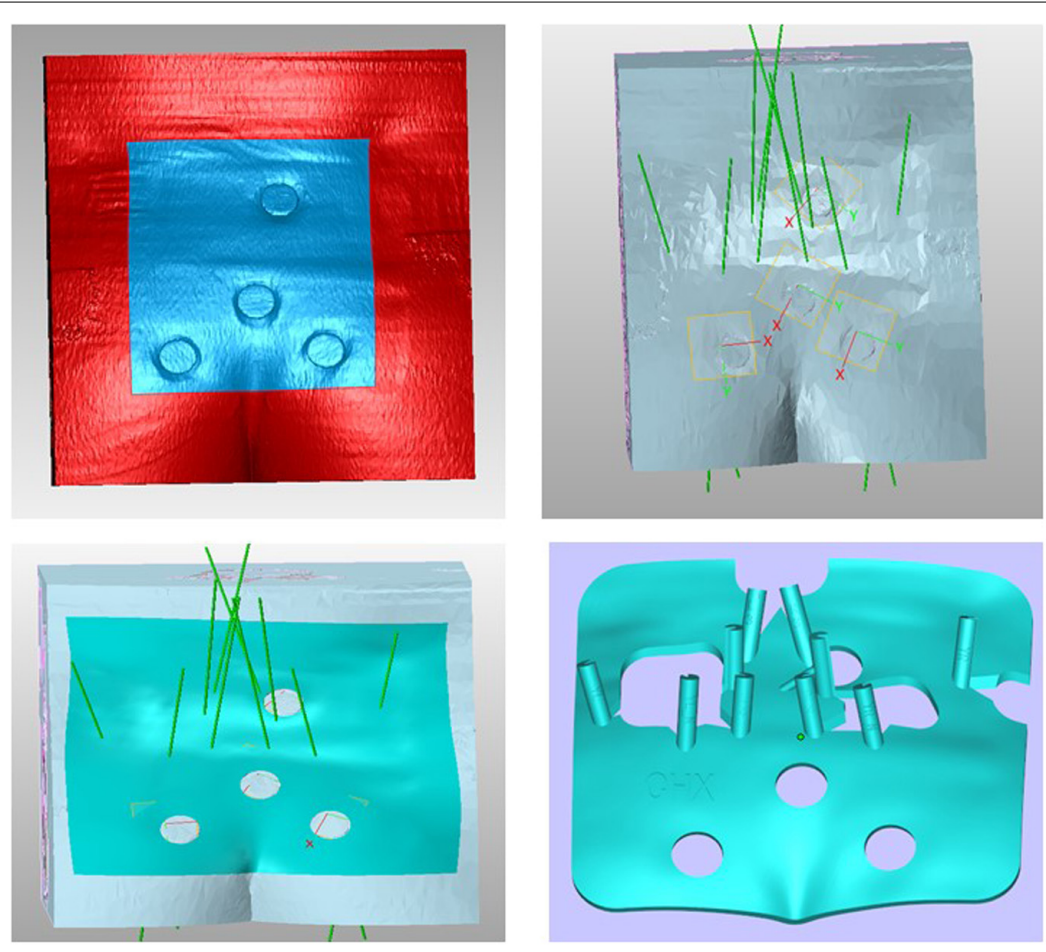

FIGURE 6 | Puncture design and modeling of 3D printing guide plate.

of symptom improvement determined whether a permanent stimulator was placed and the location of the implanted nerve was determined. A Medtronic 3889 nerve stimulation electrode was used as the nerve stimulation electrode, a Medtronic 3531 Verify stimulator was used for phase I testing, and a Medtronic 3058 nerve stimulator was used as the phase II implantable stimulator, with parameter settings of frequency $2-45 \mathrm{~Hz}$, pulse width $150-360 \mu \mathrm{s}$, and voltage of $<3 \mathrm{v}$.

\section{Statistical Method}

SPSS 20.0 software was used for statistical analysis. Measurement data are expressed as mean \pm standard deviation. $T$ test was used for pairwise comparison of measurement data, and $p<0.05$ was considered statistically significant.

\section{RESULTS}

A total of 16 patients were included in the study. All patients who underwent surgery underwent postoperative pelvic floor computed tomography to assess whether the electrode position was adapted to the pudendal nerve and sacral nerve (Figure 9). Improvement of pain, urinary frequency, and dysuria were scored on a 0 -10-point scale, where 0 represented no improvement and 10 represented complete improvement. Of a total of 16 patients, seven were male and nine were female, and their mean age was 50.56 years. There were seven patients with perineal pain, three patients with frequent urination and urgent urination, and six patients with poor urination. Five patients did not have a permanent stimulator, two patients were implanted with 

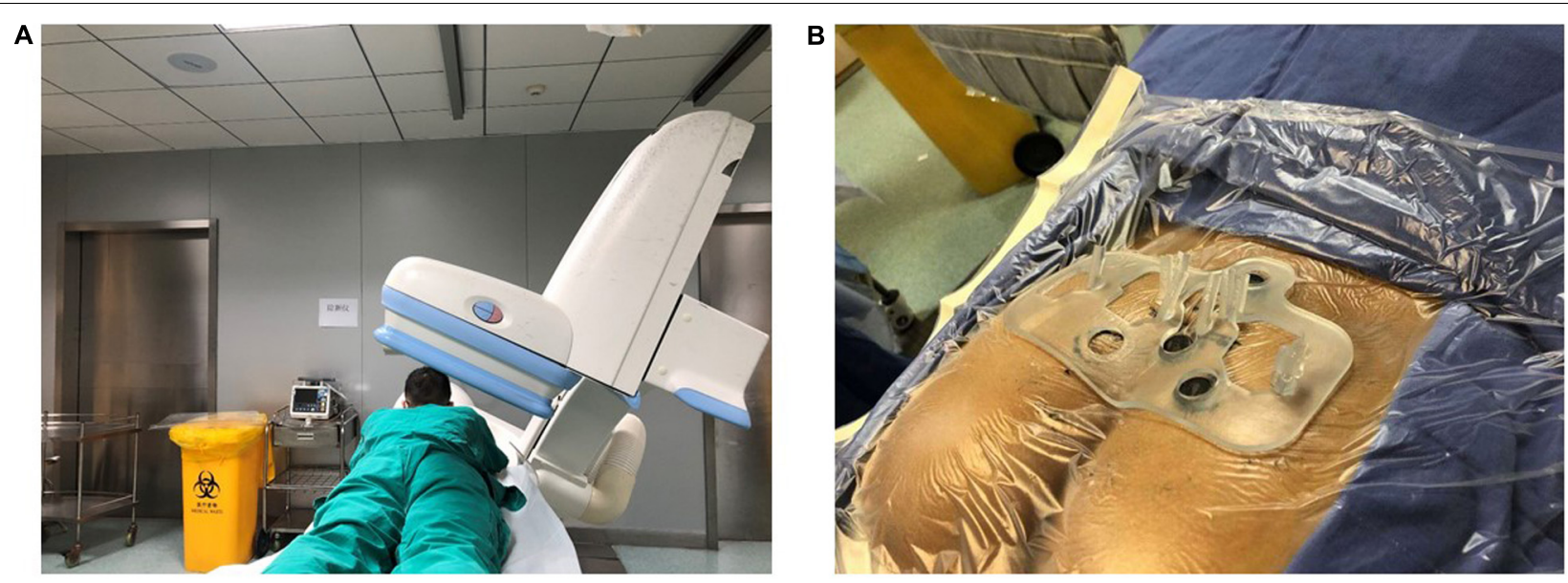

a: Intraoperative position and X-ray perspective of the patient ;

b: Intraoperative template demonstration

FIGURE 7 | Presentation of X-ray perspective and surgical template. (A) Intraoperative position and X-ray perspective of the patient; (B) Intraoperative template demonstration.
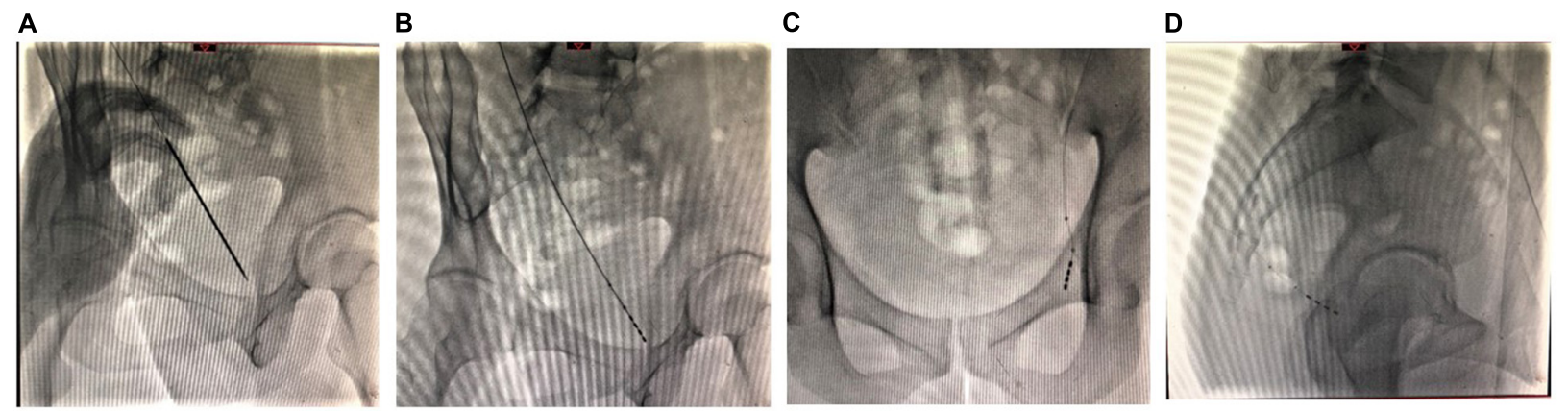
a、 b: Puncture sheath and electrode position at $45^{\circ}$ on $\mathrm{X}$-ray side; c: $\mathrm{X}$ - ray positive
perineal nerve electrode position; $\mathrm{d}: \mathrm{X}$-ray lateral perineal nerve electrode position

FIGURE 8 | Intraoperative image presentation of PNM I. (A,B) Puncture sheath and electrode position at $45^{\circ}$ on X-ray side; (C) X-ray positive perineal nerve electrode position; (D) $\mathrm{X}$-ray lateral perineal nerve electrode position.

the sacral nerve, and nine patients were implanted with the perineal nerve.

The improvement of pain in the 16 patients $(6.33 \pm 1.63$ points) was superior to the improvement in urinary frequency (3.71 \pm 1.95 points) and dysuria $(4.25 \pm 1.71$ points $)(p<0.05)$, but pudendal nerve stimulation also had an effect on urinary frequency and dysuria. No patients had any serious complications throughout the surgical treatment. Symptom scores are shown in Table 1.

\section{DISCUSSION}

Although electrical nerve stimulation is an effective treatment for neurological diseases, researchers continue to seek new neural targets to utilize in new methods in the field of neuroscience. Armstrong and Vancaillie (2016) previously described the case of a 35-year-old woman whose pain was greatly improved by sacral and pudendal nerve therapy. In a retrospective analysis, Peters et al. (2015) also reported that significant improvements in patients with pudendal neuralgia were achieved via chronic pudendal neuromodulation. These data are consistent with the preliminary data reported herein. As well as being effective for the treatment of pain, pudendal neuromodulation can also improve symptoms such as frequent urination (Wang et al., 2016, 2017). Notably, however, the pudendal nerve has no bony localization, so surgically locating it and puncturing it are problematic. For this reason the use of $3 \mathrm{D}$ printed technology to assist localization and puncture of the pudendal nerve was investigated in the present study. It is still the first time to be applied in the 

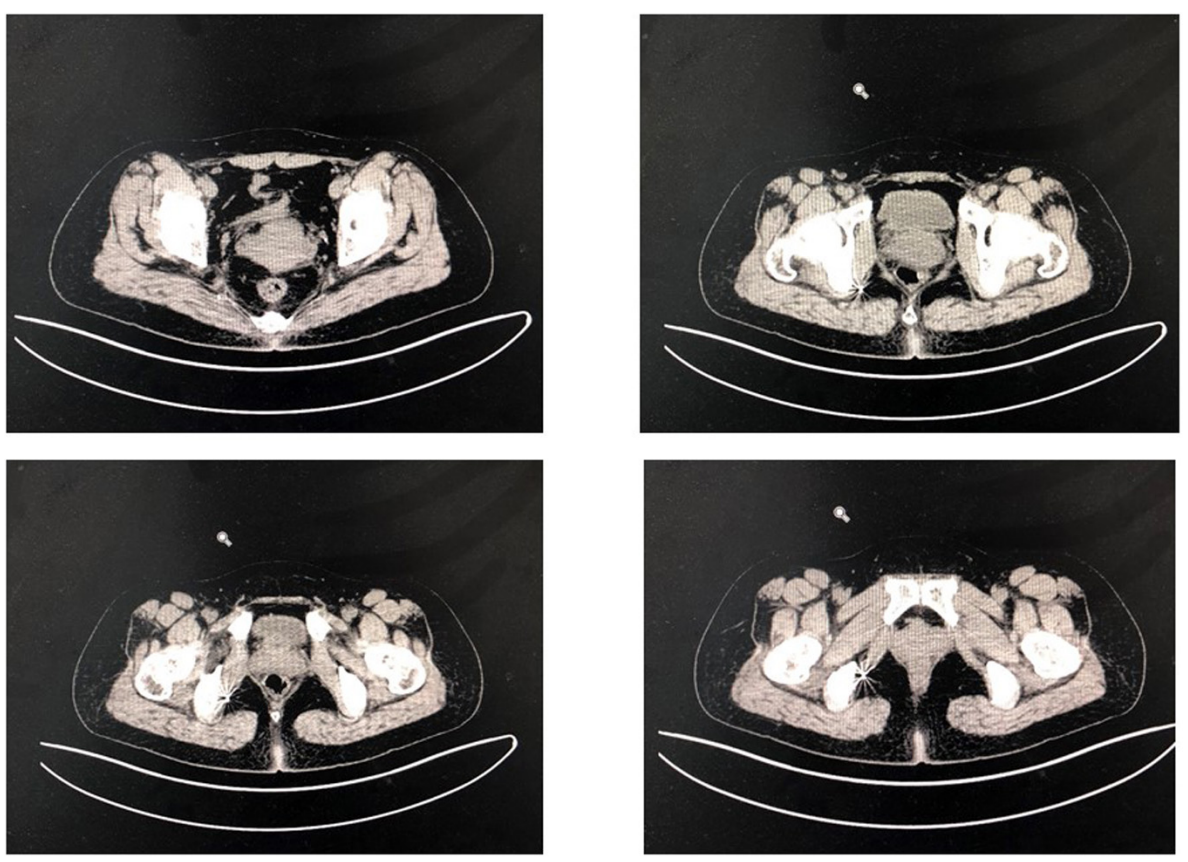

FIGURE 9 | Imaging position of electrode 1 month after operation.

TABLE 1 | The clinical situation.

\begin{tabular}{|c|c|c|c|c|c|c|c|}
\hline Serial number & Gender & Age & Main symptoms & $\begin{array}{c}\text { Transformation } \\
\text { situation }\end{array}$ & $\begin{array}{c}\text { Dysuria improves } \\
\text { symptoms }\end{array}$ & $\begin{array}{l}\text { Improved urinary } \\
\text { frequency }\end{array}$ & Pain relief* \\
\hline 1 & $\mathrm{~F}$ & 77 & Pain & NON & - & - & 2.0 \\
\hline 2 & $\mathrm{~F}$ & 54 & Pain & Pudendal nerve & - & 3.0 & 6.0 \\
\hline 3 & $M$ & 25 & Frequent urination & Pudendal nerve & - & 4.0 & - \\
\hline 4 & $\mathrm{~F}$ & 77 & Pain & Pudendal nerve & - & - & 8.0 \\
\hline 5 & $\mathrm{M}$ & 50 & Dysuria & NON & 2.0 & - & - \\
\hline 6 & $\mathrm{M}$ & 33 & Dysuria & Pudendal nerve & 6.0 & 3.0 & - \\
\hline 7 & $\mathrm{~F}$ & 65 & Pain & Pudendal nerve & - & - & 7.0 \\
\hline 8 & $\mathrm{~F}$ & 80 & Pain & Sacral nerve & - & 5.0 & 5.0 \\
\hline 9 & $\mathrm{M}$ & 41 & Dysuria & Pudendal nerve & 4.0 & - & - \\
\hline 10 & $\mathrm{~F}$ & 40 & Frequent urination & Sacral nerve & - & 5.0 & 4.0 \\
\hline 11 & $M$ & 30 & Dysuria & Pudendal nerve & 5.0 & 3.0 & - \\
\hline 12 & $\mathrm{~F}$ & 35 & Pain & NON & - & - & 3.0 \\
\hline 13 & $\mathrm{~F}$ & 44 & Pain & Pudendal nerve & - & - & 8.0 \\
\hline 14 & $\mathrm{~F}$ & 62 & Dysuria & NON & 0.0 & - & - \\
\hline 15 & M & 44 & Frequent urination & Pudendal nerve & - & 3.0 & 6.0 \\
\hline 16 & M & 52 & Dysuria & NON & 3.0 & - & - \\
\hline
\end{tabular}

*The improvement of pain, urinary frequency, and dysuria were statistically different $(P=0.05$ and $P=0.037)$.

field of nerve regulation, especially in the pelvic floor and peripheral nerve.

3D printed navigation has the following advantages: (1) It is a personalized navigation based on each individual patient's pelvic data, thus the surgery is more accurate and the electrode can better adapt to the pudendal nerve, resulting in better efficacy. (2) The surgical method draws on the traditional sacral nerve regulation surgical method, which is minimally invasive and reversible because it does not destroy surrounding tissues and nerves. (3) It is simple and convenient, and the surgeon can adapt to it by mastering the traditional sacral nerve regulation surgical method. (4) The patient does not require general anesthesia at any point during the procedure (local anesthesia is sufficient), and postoperative recovery is rapid and without serious complications. 
3D printed navigation has some limitations. MRI pudendal nerve data are difficult to obtain. Different scanning parameters are required for different brands of instruments, and in the current study $8 \mathrm{~h}$ of MRI monitoring were required to obtain the MRI scanning parameters required to perform the procedure. Lastly, 3D printed navigation is not monitored, which renders the operation susceptible to error effects.

\section{CONCLUSION}

In summary, pudendal nerve stimulation is a new therapeutic option for pelvic floor diseases. It can have beneficial effects on pain, frequent urination, and poor urination, but it may be most effective for treating pain. Utilizing a combination of autopsy data and patient-derived pelvic and nerve data, a personalized design method was developed in the current study. Neural modulation of the pudendal nerve was achieved by implanting a lead with the guidance of 3D printed navigation. Three-dimensional printing navigation can maximize the phase II conversion rate, reduce the difficulty of surgery, shorten the operation time, reduce damage to additional organs and blood vessels, and increase the accuracy of implanted electrodes, and it can be performed while the patient is awake. This surgical method is accurate, reversible, efficient, and minimally invasive.

\section{REFERENCES}

Armstrong, G. L., and Vancaillie, T. G. (2016). Combined sit-specific sacral neuromodulation and pudendal nerve release surgery in a patient with interstitial cystitis and persistent arousal. Br. Med. J. 2016:bcr2015213513. doi: 10.1136/bcr-2015-213513

Bhide, A. A., Tailor, V., Fernando, R., Khullar, V., and Alessandro Digesu, G. (2020). Posterior tibial nerve stimulation for overactive bladder-techniques and efficacy. Int. Urogynecol. J. 31, 865-870. doi: 10.1007/s00192-019-04186-3

Chen, G-q., Song, Y., Ding, L-c., Wang, J-y., Yang, Y., Wei, Z-q., et al. (2014). Chinese expert consensus on clinical application of sacral neuromodulation. Chin. J. Urol. 35, 1-5.

Expert Consensus Writing Group on Clinical Application of Sacral Neuromodulation (2017). Reprint of Chinese expert consensus on clinical application of sacral neuromodulation. Chin. J. Urol. 11, 801-804.

Han, E., Nguyen, L., Sirls, L., and Peters, K. (2018). Current best practice management of interstitial cystitis/bladder pain syndrome. Ther. Adv. Urol. 10, 197-211. doi: 10.1177/1756287218761574

Han, X., Sharma, N., Xu, Z., Scheideler, L., Geis-Gerstorfer, J., Rupp, F., et al. (2019). An in vitro study of osteoblast response on fused-filament fabricaton 3D printed PEEK for dental and cranio-maxillofacial implants. J. Clin. Med. 8:771. doi: $10.3390 /$ jcm 8060771

Jottard, K., Bonnet, P., Bruyninx, L., Ploteau, S., and De Wachter, S. (2017). The ENTRAMI technology: endoscopic transgluteal minimal invasive technique for implantation of a pudendal electrode under full visual control: a cadaver study. Neurourol. Urodyn. 38, 130-134. doi: 10.1002/nau.23850

Kessler, T. M., La Framboise, D., Trelle, S., Clare, J., Fowler, C. J., Kiss, G., et al. (2010). Sacral neuromodulation for neurogenic lower urinary tract dysfunction: systematic review and meta-analysis. Eur. Urol. 58, 865-874. doi: 10.1016/j. eururo.2010.09.024

Konschake, M., Brenner, E., Moriggl, B., Hörmann, R., Bauer, S., Foditsch, E., et al. (2017). New laparoscopic approach to the pudendal nerve for neuromodulation based on an anatomic study. Neurourol. Urodyn. 36, 1069-1075. doi: 10.1002/ nau. 23090

Lal, H., and Patralekh, M. K. (2018). 3D printing and its applications in orthopaedic trauma: a technological marvel. J. Clin. Orthop. Treuma 9, 260-268. doi: 10. 1016/j.jcot.2018.07.022

\section{DATA AVAILABILITY STATEMENT}

The original contributions presented in the study are included in the article/supplementary material, further inquiries can be directed to the corresponding author.

\section{ETHICS STATEMENT}

The studies involving human participants were reviewed and approved by the Renji Hospital, Shanghai Jiao Tong University School of Medicine. The patients/participants provided their written informed consent to participate in this study. Written informed consent was obtained from the individual(s) for the publication of any potentially identifiable images or data included in this article.

\section{AUTHOR CONTRIBUTIONS}

YG: data curation and original manuscript draft preparation. TL: software operation and data validation. CJ: patients pay a return visit. JL: supervision and manuscript writing, reviewing, and editing. All authors contributed to the article and approved the submitted version.

Marinkovic, S. P., Miller, B., Hughes, S., Marinkovic, C., and Gillen, L. (2015). Sucessful bilateral pudendal neuromodulation to treat male detrusor areflexia following severe pubic symphysis fracture, a case report. BMC Urol. 15:115. doi: 10.1186/s12894-015-0108-4

Morrison, T. J., Sefton, E., Marquez-Chin, M., Popovic, M. R., Morshead, C. M., and Naguib, H. E. (2019). A 3D printed device for low cost neural stimulation in mice. Front. Neurosci. 13:784. doi: 10.3389/fnins.2019. 00784

Peters, K. M., Killinger, K. A., Jaeger, C., and Chen, C. (2015). Pilot study exploiting chronic pudendal neuromodulation as a treatment option for pain associated with pudendal neuralgia. Low. Urin. Tract Symptoms 7, 138-142. doi: 10.1111/ luts. 12066

Pirro, N., Sielezneff, I., Le Corroller, T., Ouaissi, M., Sastre, B., and Champsaur, P. (2009). Surgical anatomy of the extrapelvic part of the pudendal nerve and its applications for clinical practice. Surg. Radiol. Anat. 31, 769-773. doi: 10.1007/s00276-009-0518-7

Reitz, A., Gobeaux, N., Mozer, P., Delmas, V., Richard, F., and Chartier-Kastler, E. (2007). Topographic anatomy of a new posterior approach to the pudendal nerve for stimulation. Eur. Urol. 51, 1350-1356. doi: 10.1016/j.eururo.2006. 10.004

Salima, I., Chartier-Kastler, E., Perrouin-Verbe, M. A., Rose-Dite-Modestine, J., Denys, P., and Phé, V. (2017). Long-term functional outcomes of S3 sacral neuromodulation for the treatment of ldiopathic overactive bladder. Neuromodulation 20, 825-829. doi: 10.1111/ner.12696

Schmidt, R. A., Bruschini, H., and Tanagho, E. A. (1979). Sacral root stimulation in controlled micturition. Peripheral somatic neurotomy and stimulated voiding. Invest. Urol. 17, 130-134.

Wang, S., Lv, J., Feng, X., and Lv, T. (2017). Efficacy of electrical pudendal nerve stimulation versus transvaginal electrical stimulation in treating female idiopathic urgency urinary incontinence. J. Urol. 197, 1496-1501. doi: 10.1016/ j.juro.2017.01.065

Wang, S., Lv, J., Feng, X., Wang, G., and Lv, T. (2016). Efficacy of electrical pudendal nerve stimulation in treating female stress incontinence. Urology 91, 64-69. doi: 10.1016/j.urology.2016.02.027

Yinjun, G. U., Tinging, L. V., Weilin, F. A. N. G., Qibo, F. U., Jing, L. E. N. G., Wei, X. U. E., et al. (2016). Evaltion of 3D printing in the application of the sacral nerve regulation technique. J. Clin. Urolol. 31, 1057-1063. 
Zhang, J., Zhang, P., Wu, L., Su, J., Shen, J., Fan, H., et al. (2018). Application of an individualized and reassemblable 3D printing navigation template for accurate puncture during sacral neuromodulation. Neurourol. Urodyn. 37, 2776-2781. doi: $10.1002 /$ nau. 23769

Zhang, P., Wang, J. Y., Zhang, Y., Liao, L., Lv, J. W., Ling, Q., et al. (2019). Results of sacral neuromodulation therapy for urinary voiding dysfuncton: five-year experience of a retrospective, multicenter study in China. Neuromodulation 22, 730-737. doi: $10.1111 /$ ner.12902

Zhang, P., Zhang, J-z., Wu, L-y., Zhang, Z-h., and Zhang, X-d. (2017). Clinical study of SNM on non-neurogenic, nonobstructive dysuria. Chin. J. Urol. 38, 806-810.
Conflict of Interest: The authors declare that the research was conducted in the absence of any commercial or financial relationships that could be construed as a potential conflict of interest.

Copyright $\odot 2021 \mathrm{Gu}$, Lv, Jiang and Lv. This is an open-access article distributed under the terms of the Creative Commons Attribution License (CC BY). The use, distribution or reproduction in other forums is permitted, provided the original author(s) and the copyright owner(s) are credited and that the original publication in this journal is cited, in accordance with accepted academic practice. No use, distribution or reproduction is permitted which does not comply with these terms. 